\title{
Study on mechanical properties of polyethylene with chain branching in atomic scale by molecular dynamics simulation
}

\section{Lijuan Liao, Chenguang Huang \& Changyu Meng}

To cite this article: Lijuan Liao, Chenguang Huang \& Changyu Meng (2018): Study on mechanical properties of polyethylene with chain branching in atomic scale by molecular dynamics simulation, Molecular Simulation, DOI: 10.1080/08927022.2018.1471690

To link to this article: https://doi.org/10.1080/08927022.2018.1471690

曲 Published online: 10 May 2018.

Submit your article to this journal $\llbracket$

山 Article views: 8

Q View related articles $\square$

View Crossmark data 〔 


\title{
Study on mechanical properties of polyethylene with chain branching in atomic scale by molecular dynamics simulation
}

\author{
Lijuan Liao $^{\mathrm{a}} \mathbb{D}$, Chenguang Huanga, and Changyu Meng ${ }^{\mathrm{a}, \mathrm{b}}$ \\ ${ }^{a}$ Key Laboratory for Mechanics in Fluid Solid Coupling Systems, Institute of Mechanics, Chinese Academy of Sciences, Beijing, China; bschool of \\ Engineering Science, University of Chinese Academy of Sciences, Beijing, China
}

\begin{abstract}
The effects of length and content of chain branching on the mechanical properties of polyethylene (PE) in atomic scale were examined by molecular dynamics (MD) simulations. Methyl-, ethyl- and butylgroups were adopted as branched chains to distribute along PE backbones. Plastic flow deformation was captured by providing a uniaxial tensile loading at a given strain rate, which shows the characteristic of rate dependence. Current results are in reasonable agreements with existing experimental data. The statistical results show that the longer length of chain branching induces lower equilibrium density and higher yield strength of branched PE. In addition, higher content of chain branching brings higher equilibrium density and lower yield strength of branched PE. It is assumed that the distribution of dihedral angles influences the deformation of PE definitely. The non-bond interactions contribute to the load-bearing capacity of PE largely. Branched PE shows big differences on mechanical behaviours comparing with the linear one. Chain branching distribution also greatly affects the performance of PE, which needs a further discussion.
\end{abstract}

ARTICLE HISTORY

Received 14 September 2017

Accepted 28 April 2018

\section{KEYWORDS}

Chain branching; polyethylene (PE); mechanical properties: molecular dynamics (MD) simulation; atomic scale

\section{Introduction}

Polyethylene (PE) is one of the most common polymers used widely in industries. Obtaining the comprehensive properties and corresponding mechanisms is helpful to expand its use in a broader scope. Actually, the versatility in different forms of $\mathrm{PE}$ is significantly affected by its molecular structure [1,2], which is a vital factor governing physical performance [3].

PE is classified according to density and degree of molecular branching $[1,2,4,5]$. With highly branched molecular structure, low-density PE (LDPE) is soft, tough and flexible, which is characterised by loose instead of packing well into crystallites. On the contrary, with the promotion of linearity degree, such as high-density PE (HDPE) or even ultra-high molecular weight PE (UHMWPE), the ability of being tightly packed improves greatly. While, with a large number of short chain branching, the properties of linear LDPE (LLDPE) show different aspects compared with LDPE or HDPE $[1,4]$. These phenomena attract broad research interests in the effects of molecular structures with blend of short and long branched chains on the properties of PE, such as rheology $[2,6,7]$, deformation mechanisms [3,8], processability [9] and other mechanical properties $[1,3,4,10-15]$. Liu and Baker [11] described that these branched chains play the similar role as the rubber particles in a rubber-toughened blend which gives rise to the concept of a 'one copolymer blend.'

The essential properties of PE are mainly obtained through experiments. A few computational models based on continuum mechanics depending on metal materials were also established to provide phenomenological understanding [16-18]. The mechanism of amorphous complex structures is still difficult to be grasped using continuum mechanical evaluation only. The essential effects of microstructures on mechanical behaviours of polymers should be clarified firstly to provide atomistic descriptions.

Molecular dynamics (MD) simulation is useful to understand the complex behaviour of polymers in microscale $[1,8,19,20]$, which indicates the intrinsic mechanism for macroscale mechanical behaviours without phenomenological assumption. Correspondingly, various force fields, such as AMBER [21,22], CHARMM [22], OPLS [23,24], PCFF [25] and COMPASS [26], have been developed to govern the intra- and inter-molecular interactions using potential energies. Moyassari et al. [13] developed a Monte Carlo simulation method on explicitly branched PE. They obtained that the concentration of inter-lamellar connections increases significantly by introducing a few short branches to an unbranched PE. In addition, the average end-toend distance of a short chain with same molar mass increases as branching increases.

The microstructure of polymer plays a significant role in mechanical responses, which are essential to affect its reliability and design-potential for further applications. Consequently, atomic-scale PE with three types of branched chains was examined by MD. The size-dependence on chain length and number of chains were evaluated. The characteristics of the molecular structures of linear and branched PE at equilibrium were described. The effects of branching on the dynamic mechanical performances were analyzed. The adopted method was validated 
by comparing with the available experimental results. Finally, the practicability of adopting linear PE instead of branched one in simulations to describe the properties was also discussed.

\section{Simulation methodology}

\subsection{Molecular structures of PE chains}

As a mixture of similar polymer of ethylene $\left(\mathrm{C}_{2} \mathrm{H}_{4}\right)$, pure linear $\mathrm{PE}$ chains are rarely in nature. Instead, various $\mathrm{PE}$ with branching are common as functional materials [4]. Three typical branching, such as methyl $-\left[-\mathrm{CH}_{3}\right]$, ethyl $-\left[-\mathrm{CH}_{2}-\mathrm{CH}_{3}\right]$ and butyl $-\left[-\mathrm{CH}_{2}-\right.$ $\mathrm{CH}_{2}-\mathrm{CH}_{2}-\mathrm{CH}_{3}$ ] group, were selected to discuss their effects on properties of PE. The branch length $l$ equals to the number of carbon atoms contained in each branched chain. Accordingly, the branch length $l$ of methyl, ethyl and butyl group is one, two and four, respectively. These groups are located along the backbone $\left(-\left[-\mathrm{CH}_{2}-\mathrm{CH}_{2}-\right]_{m}-\right)$ of $\mathrm{PE}$, where the number of ethylene monomer $m$ represents the length of backbone. Linear PE chains with monomers $-\left[-\mathrm{CH}_{2}-\mathrm{CH}_{2}-\right]_{n}-$ were also modelled without any branching as a reference. The determination of the length $n$ of a single chain will be described in Section 3.1. As a result, $n$ was chosen as $25\left(\mathrm{C}_{50} \mathrm{H}_{102}=152\right.$ atoms in a single chain $)$ in the present study. Fragments of atomic-scale models are shown in Figure 1 (all visualizations in this paper are implemented via VMD [27]). Different categories were organized to examine the effects of type, length and content of chain branching as listed in Table 1. It should be noted that $n_{\mathrm{BC}}$ is the number of branched chains $(\mathrm{BC})$. In addition, $m_{\mathrm{BC}}$ indicates the total number of carbon atoms in the branched chains to describe the content, which equals to the product of $l$ and $n_{\mathrm{BC}}$. As mentioned above, $2 n$ is the total number of carbon atoms in a single PE chain, whose value is fifty in this paper. And, $2 m$ is the total number of carbon atoms in a backbone. Therefore, two relations of $l \times n_{\mathrm{BC}}+2 m=m_{\mathrm{BC}}+2 m=2 n$ and $m_{\mathrm{BC}} / 2 n+2 m / 2 n=1$ can be deduced.

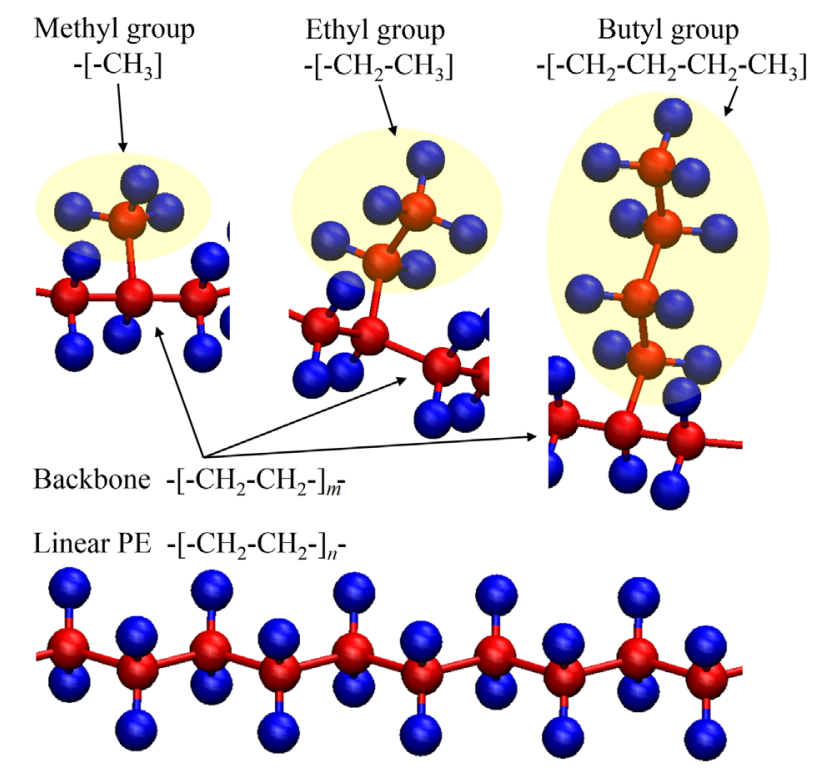

red particle: carbon atom $(\mathrm{C})$, blue particle: hydrogen atom $(\mathrm{H}))$

Figure 1. (Colour online) Fragments of atomic-scale models for chain branching group along a backbone and a linear PE chain.
Table 1. PE systems with various branched chains.

\begin{tabular}{lllrccccc}
\hline PE Type & Branching & $I$ & $n_{\mathrm{BC}}$ & $m_{\mathrm{BC}}$ & $2 m$ & $m_{\mathrm{BC}} / 2 n$ & $2 m / 2 n$ & $1 / 2 m$ \\
\hline Linear & $\times$ & 0 & 0 & 0 & 50 & 0 & 1 & 0 \\
Branched & Methyl & 1 & 8 & 8 & 42 & 0.16 & 0.84 & 0.02 \\
& Ethyl & 2 & 4 & & & & & 0.05 \\
& Butyl & 4 & 2 & & & & & 0.10 \\
& Methyl & 1 & 16 & 16 & 34 & 0.32 & 0.68 & 0.03 \\
& Ethyl & 2 & 8 & & & & & 0.06 \\
& Butyl & 4 & 4 & & & & & 0.12 \\
& Methyl & 1 & 24 & 24 & 26 & 0.48 & 0.52 & 0.04 \\
& Ethyl & 2 & 12 & & & & & 0.08 \\
& Butyl & 4 & 6 & & & & & 0.15 \\
\hline
\end{tabular}

Notes: $/$ is the number of carbon atoms contained in each branched chain (methyl group: $I=1$, ethyl group: $I=2$, butyl group: $I=4$ ).

$n_{B C}$ is the number of branched chains in a single PE chain.

$m_{\mathrm{BC}}$ is the total number of carbon atoms contained in branched chains, which equals to the product of $/$ and $n_{\mathrm{BC}}$.

$2 m$ is the total number of carbon atoms contained in a single backbone.

$2 n$ is the total number of carbon atoms contained in a single PE chain, which is 50 in this paper.

$1 \times n_{\mathrm{BC}}+2 m=m_{\mathrm{BC}}+2 m=2 n$ and $m_{\mathrm{BC}} / 2 n+2 m / 2 n=1$.

A single PE chain with branching was modelled (performed in Moltemplate [28]) by randomly seeding the given branching type with given numbers and corresponding methylene monomers $-\left[-\mathrm{CH}_{2}-\right]-$ as the backbone, which is connected with a methyl group $-\left[-\mathrm{CH}_{3}\right]$ both at head and tail. Given number (marked as $n_{r}$ ) of PE chains were packed into a cubic box with periodic boundary conditions in all three orthogonal directions. Here, $100 \mathrm{PE}$ chains were included in a single box, which was decided by the discussions in Section 3.1. Three samples were established with a unique configuration for each case listed in Table 1 to carry out a statistical analysis.

\subsection{Force field selection}

The OPLS All-Atom Force Field was chosen to describe the intraand inter-molecular interactions, in which the expressions of potential energies are listed below [24].

Bond stretching:

$$
E_{\text {bond }}=\sum_{\text {bonds }} K_{r}\left(r-r_{e q}\right)^{2}
$$

Angle bending:

$$
E_{\text {angle }}=\sum_{\text {angles }} K_{\theta}\left(\theta-\theta_{e q}\right)^{2}
$$

Torsion:

$$
\begin{aligned}
E(\phi)= & \frac{V_{1}}{2}[1+\cos (\phi+f 1)] \\
& +\frac{V_{2}}{2}[1-\cos (2 \phi+f 2)] \\
& +\frac{V_{3}}{2}[1+\cos (3 \phi+f 3)]
\end{aligned}
$$

Non-bonded:

$$
\begin{gathered}
E_{a b}=\sum_{i}^{\text {ona on }} \sum_{j}^{\text {on }}\left[4 \varepsilon_{i j}\left(\sigma_{i j}^{12} / r_{i j}^{12}-\sigma_{i j}^{6} / r_{i j}^{6}\right)+q_{i} q_{j} e^{2} / r_{i j}\right] f_{i j} \\
f_{i j}=0.5 \text { if } i, j \text { are } 1,4 \text {;otherwise, } f_{i j}=1.0
\end{gathered}
$$


where $K_{r}$ and $K_{\theta}$ are the stiffness coefficients of bond stretching and angle bending potentials, respectively. In addition, $r_{e q}$ and $\theta_{e q}$ are the equilibrium bond length and angle, respectively. Furthermore, $V_{i}(i=1,2,3)$ is the coefficient in the Fourier series and $f(i=1,2,3)$ is phase angle.

Bond stretching, angle bending and dihedral torsion compose the valence terms. The non-bond terms consist of the van der Waals (12-6 Lennard-Jones potential with a cutoff distance of $10.0 \AA$ ) and the Coulombic forces by particle-particle-particle-mesh approach.

\subsection{Relaxation process}

The simulations were carried out by Large-scale Atomic/ Molecular Massively Parallel Simulator (LAMMPS) [29]. Initial configuration experiences a relaxation with a time step 0.5 fs to reach equilibrium as follows:

Step 1. Energy minimisation was performed to adjust atom coordinates firstly.

Step 2. Langevin thermostat at $3000 \mathrm{~K}$ for $1 \mathrm{~ns}$ was applied to perform Brownian dynamics.

Step 3. Further relaxation ran under NVT ensemble at $300 \mathrm{~K}$ for 200 ps using Nose-Hoover method.

Step 4. An annealing process ranging from 300 to $600 \mathrm{~K}$ with 3 cycles at a rate of $100 \mathrm{~K} / 200$ ps by NPT ensemble under atmospheric pressure (1 atm) using Nose-Hoover method was carried out for 3.6 ns to remove residual stress and obtain final equilibrium.

A snapshot of branched $\mathrm{PE}$ with four butyl groups at equilibrium is shown in Figure 2 (cell size $\approx 54 \AA \times 52 \AA \times 52 \AA$ ), which shows a high degree of amorphous PE chains.

red particle: carbon atom $(\mathrm{C}), 0$ blue particle: hydrogen atom $(\mathrm{H}))$
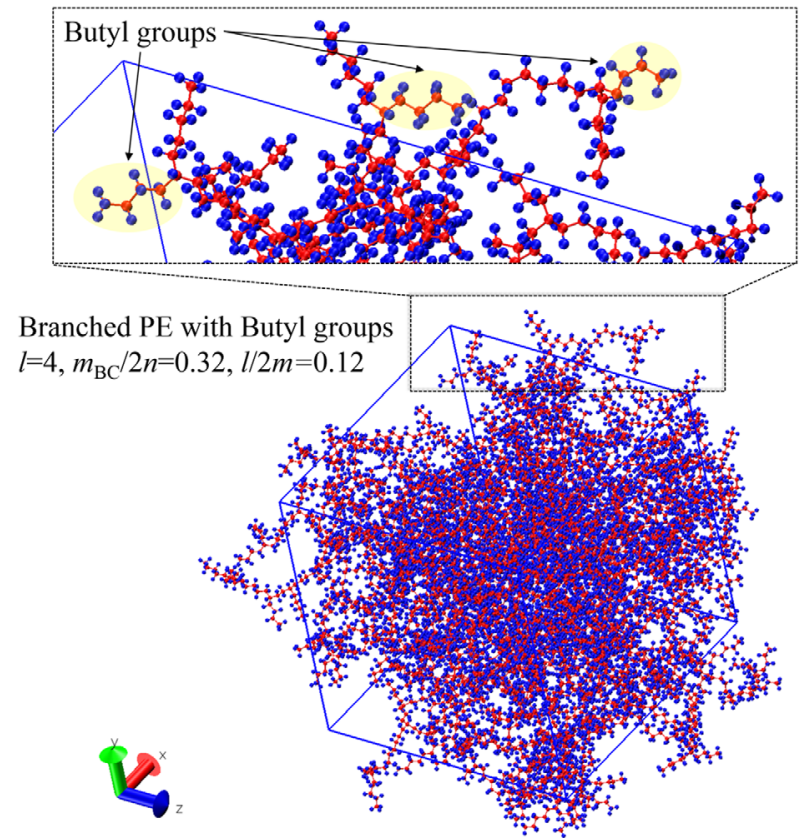

Figure 2. (Colour online) A sample of branched PE with butyl groups at equilibrium.

\section{Results and analysis}

\subsection{Determination of system size}

Three linear PE systems $\mathrm{S} i(i=1,2,3)$ with different sizes (denoted as $n \times n_{r}$ ) in chain length $(n)$ and number of chains $\left(n_{r}\right)$ were created, which were listed in Table 2. Three samples were built for each system to carry out the statistical analysis.

The size-dependence of the density at equilibrium was examined at $300 \mathrm{~K}$, which is the average value of the last $4 \times 10^{5}$ time steps in the relaxation. The densities at equilibrium of linear PE systems (S1: $0.79 \pm 3.18 \mathrm{E}-4 \mathrm{~g} / \mathrm{cc}, \mathrm{S} 2: 0.80 \pm 1.23 \mathrm{E}-3 \mathrm{~g} / \mathrm{cc}, \mathrm{S} 3$ : $0.79 \pm 5.84 \mathrm{E}-4 \mathrm{~g} / \mathrm{cc}$ ) fall into the range of $0.79-0.80 \mathrm{~g} / \mathrm{cc}$, which consist with the value $0.80 \mathrm{~g} / \mathrm{cc}$ reported previously [19]. It can be found that the variation of density at equilibrium with respect to the size of linear PE system is slight.

In addition, the glass transition temperatures $\left(T_{g}\right)$ of PE systems, as the vital factor to characterise the thermal property, were calculated according to temperature-dependence of density as shown in Figure 3. The system at equilibrium experienced an annealing process, by heating up to $500 \mathrm{~K}$ and then cooling down to $0 \mathrm{~K}$ with a rate of $20 \mathrm{~K} / 20 \mathrm{ps}$. Under atmospheric pressure (1 atm), a simulation under NPT ensemble with NoseHoover thermostatting method was performed. As plotted in Figure 3, each point is the average value of $4 \times 10^{4}$ time steps results. Inflection points can be seen in the bi-linear fittings, which indicate the transition from glassy to rubbery state of polymer [30,31]. The average values of $T_{g}$ for linear PE systems were

Table 2. Three linear PE systems with different sizes.

\begin{tabular}{lccc}
\hline No. & $n \times n_{r}$ & Total number of atoms & $\begin{array}{c}\text { Cell size at equilibrium } \\
(x \times y \times z)\left(\AA^{3}\right)\end{array}$ \\
\hline S1 & $25 \times 100$ & 15,200 & $(54.12 \pm 0.12) \times(51.91 \pm$ \\
& & & $0.12) \times(51.91 \pm 0.12)$ \\
S2 & $100 \times 100$ & 60,200 & $(84.34 \pm 0.05) \times(82.70 \pm$ \\
& & & $0.05) \times(82.70 \pm 0.05)$ \\
S3 & $25 \times 400$ & 60,800 & $(84.80 \pm 0.12) \times(83.15 \pm$ \\
& & & $0.12) \times(83.15 \pm 0.12)$ \\
\hline
\end{tabular}

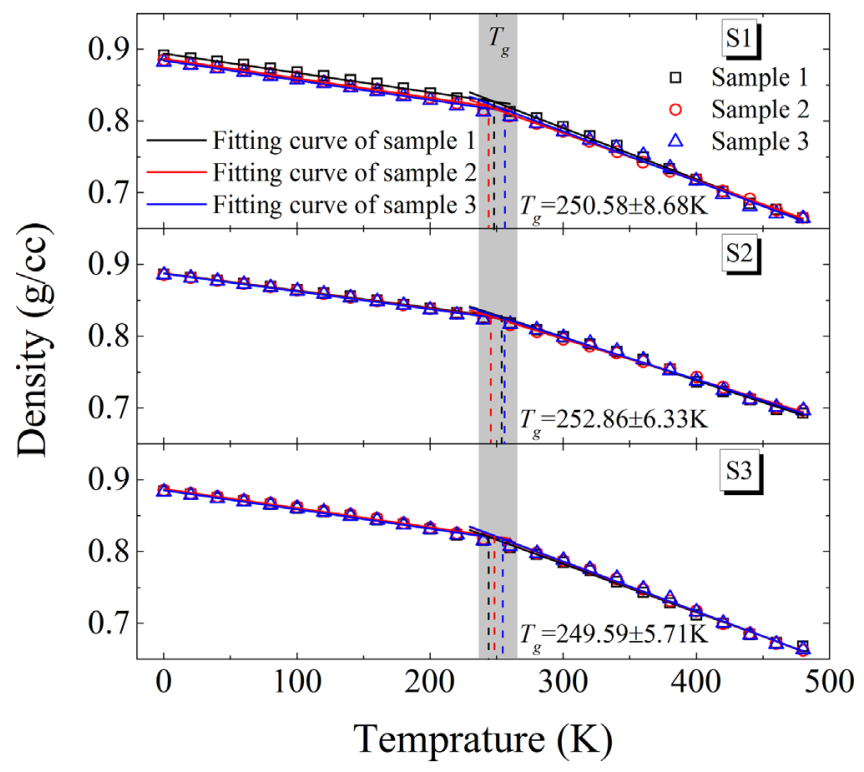

Figure 3. (Colour online) Variations of density with respect to temperature for each linear PE system. 
evaluated as $250 \mathrm{~K}$ approximately, which reasonably agree with the range reported from 220 to $250 \mathrm{~K}$ in previous MD simulations [32] and from 155 to $252 \mathrm{~K}$ in experiments [33].

The predicted density at equilibrium and $T_{g}$ are not sensitive to the system size discussed in the present study. The similar results were also observed in the studies by Hossain et al. [8] (with PE united atoms (UA, one UA represents one monomer -[- $\left.\mathrm{CH}_{2}{ }^{-}\right]$-) model size $n \times n_{r}=100 \times 200,1000 \times 20,100 \times 2000$ and $1000 \times 200$ ) and Kulmi et al. [19] (with UA model length of a single chain $n=50$ and 250). Thus, the size of $S 1$ was adopted in the present simulations to take calculation accuracy and efficiency into account simultaneously, where $n=25$ and $n_{r}=100$, respectively.

\subsection{Characteristics at equilibrium}

Statistical calculations on three samples of each case listed in Table 1 were carried out. The variations of temperature $T$ and total energy $E_{\text {total }}$ of branched PE with six butyl groups during the last 150 ps of relaxation are illustrated in Figure 4 as an example. The solid line and the shading are the average value and error band for each parameter, respectively. The temperature of the system was controlled at $300 \mathrm{~K}$ steadily. In addition, $E_{\text {total }}$ converges to a constant value with subtle fluctuation at the end of relaxation. It can be assumed that the relaxation mentioned in Section 2.3 is acceptable to reach the equilibrium.

Initial configuration of polymer chains at equilibrium plays a significant role in mechanical properties of polymers in molecular simulations. The branching parameter $g_{\mathrm{g}}$, as the best quantity to characterise the overall dimension of branched chain, is equal to the ratio of average mean square radius of gyration for branched chain $R_{\mathrm{gb}}{ }^{2}$ to average mean square radius of gyration for linear chain $R_{\mathrm{gl}}^{2}$ [34]. Figure 5 illustrates the variation of $g_{\mathrm{g}}$ with respect to branch length $l$ and content $m_{\mathrm{BC}} / 2 n$. The average value $g_{\mathrm{g}}$ of branched $\mathrm{PE}$ is less than one as a result of more congestion around the mass centre of monomers with branching. This identifies that the equilibrium configurations of branched $\mathrm{PE}$ are reasonable for performing further studies. In addition,

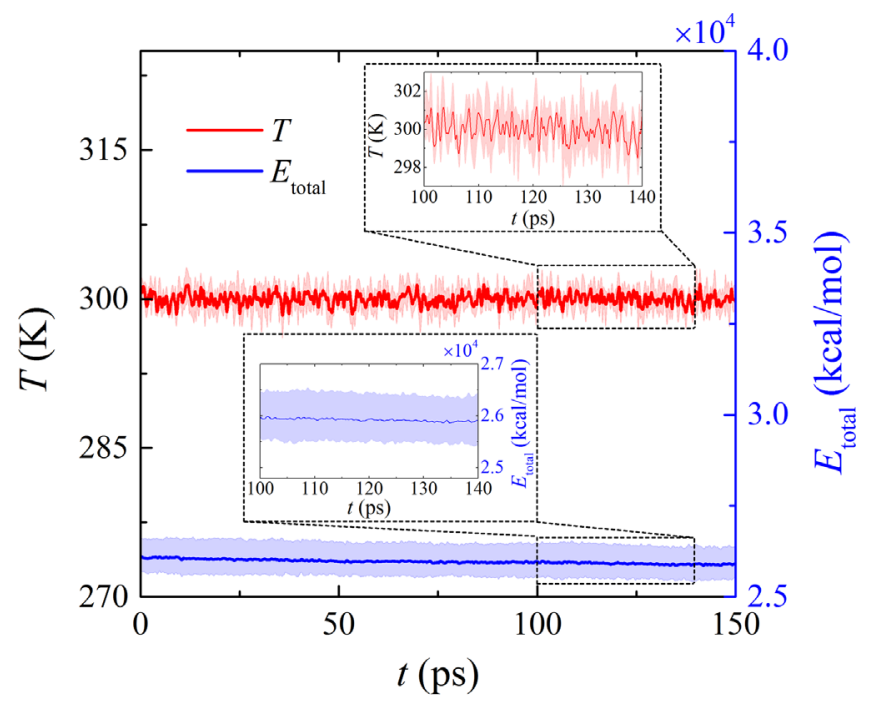

Figure 4. (Colour online) Variations of temperature and total energy of branched PE with six butyl groups at the end of relaxation.

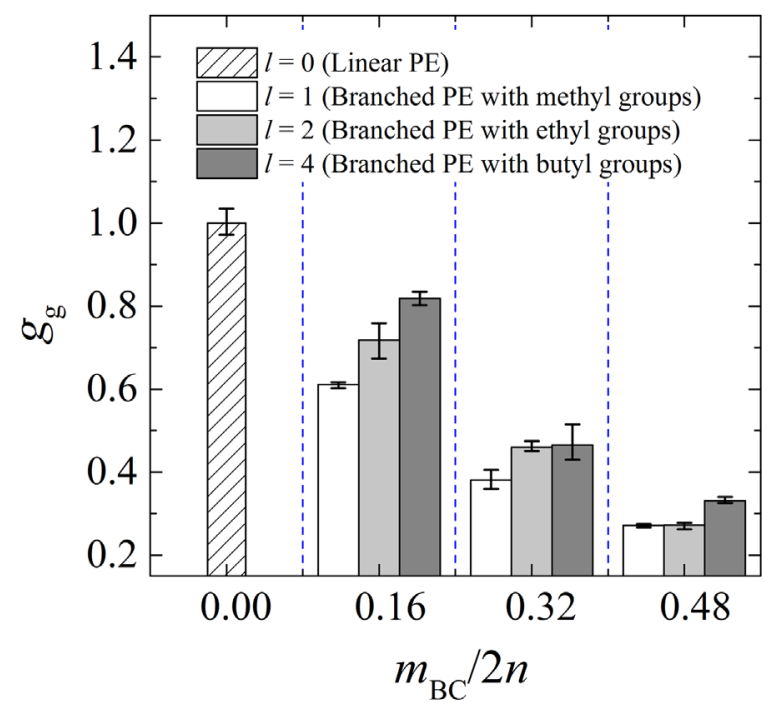

Figure 5. (Colour online) Branching parameters $g_{g}$ of PE systems at equilibrium.

$g_{\mathrm{g}}$ of branched systems increases as $l$ increases at a given branch content. An inversely proportional relationship of $g_{\mathrm{g}}$ and $m_{\mathrm{BC}} / 2 n$ is observed with a given branch length.

Average density of PE system (with and without branching) finally equilibrated at $300 \mathrm{~K}$ ranges from 0.78 to $0.82 \mathrm{~g} / \mathrm{cc}$, which is close to the value $0.80 \mathrm{~g} / \mathrm{cc}[35]$ and $0.85 \mathrm{~g} / \mathrm{cc}[36,37]$ adopted in the previous MD simulations. Figure 6 plots the variations of equilibrium density. Except for linear PE, the average value of equilibrium density is inversely proportional to branch length $l$ with a given branch content $m_{\mathrm{BC}} / 2 n$. In addition, the effect of branch length $l$ on the equilibrium density becomes more significant for higher degree of branching accompanied with shorter backbone. Equilibrium density is in proportion to branch content $m_{\mathrm{BC}} / 2 n$ with a given branch length $l$. However, the monotonicity of equilibrium density and branch content $m_{\mathrm{BC}} / 2 n$ is not strict for PE with butyl groups.

\subsection{Characteristics under tensile loadings}

The tensile deformation of PE at equilibrium was simulated by providing a uniaxial tensile loading at a given strain rate while keeping zero-pressure conditions for other two directions of simulation cell $[8,38,39]$. NPT MD simulations were run by NoseHoover thermostatting method in tensile process.

Strain rates $\dot{\varepsilon}=10^{10}, 10^{9}$ and $10^{8} / \mathrm{s}$ were adopted to examine the strain-stress relation and rate-dependence characteristic. The temperature was chosen as $240 \mathrm{~K}$, which is below $T_{g}(250.58 \pm$ $8.68 \mathrm{~K}$ for linear PE as evaluated in Figure 3), to examined the responses of linear PE systems at glassy state. The stress-strain evolution subjected to a tensile loading with a given strain rate for a single sample of linear PE was described in Figure 7. Classical four stages, including elastic, yield, soften and strain hardening regimes, can be observed $[4,8]$. The value of the stress at the global yield point is denoted as the yield strength $\sigma_{y}$. It varies with respect to strain rate monotonically $[4,8]$, which shows rate-dependence.

Figure 8 summarises the yield strengths of linear and branched PE systems subjected to a tensile loading with $\dot{\varepsilon}=10^{10} / \mathrm{s}$ at $240 \mathrm{~K}$. 


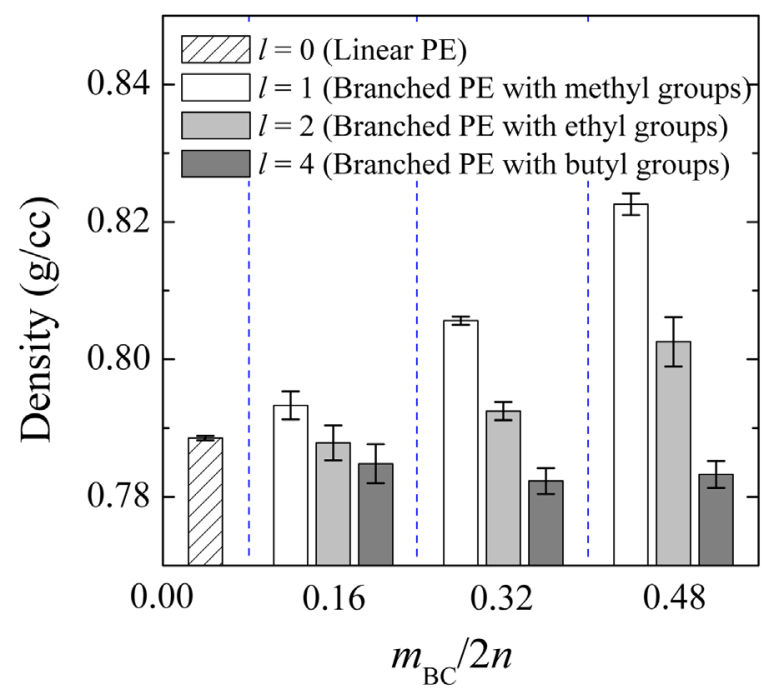

Figure 6. (Colour online) Densities of PE systems at equilibrium.

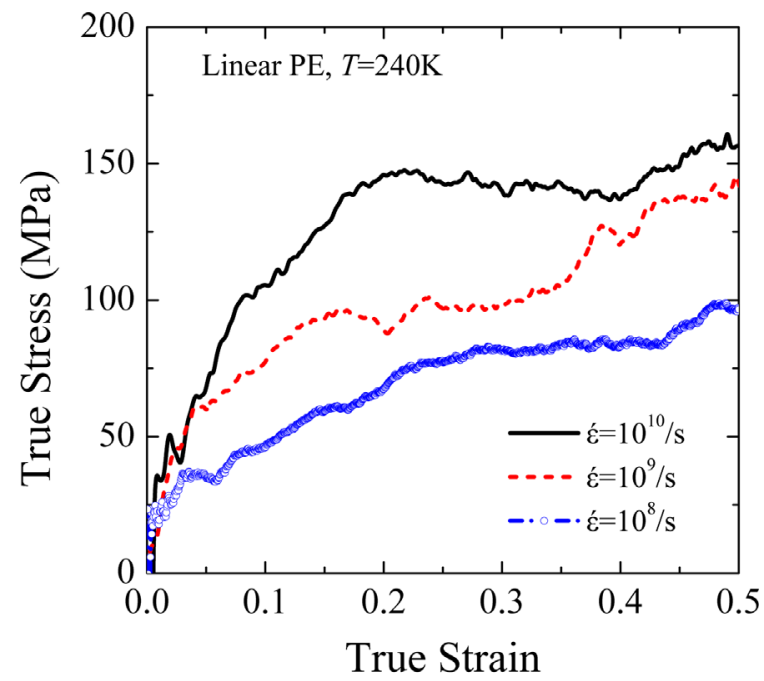

Figure 7. (Colour online) Strain-stress evolution of linear PE with respect to strain rate at $240 \mathrm{~K}$.

Regardless of linear PE, the average $\sigma_{y}$ of branched PE and branch length $l$ are directly related when branch content $m_{\mathrm{BC}} / 2 n$ remains constant. An inverse relationship between the average $\sigma_{y}$ and branch content $m_{\mathrm{BC}} / 2 n$ appears in branched $\mathrm{PE}$ with a given branch length $l$.

\subsection{Comparisons between current simulations and existing experimental data in literatures}

\subsubsection{Equilibrium density}

Liu and Baker [11] discussed the effects of branch length on impact properties of PE experimentally. They selected three commercial LLDPE resins with similar molecular weight, molecular weight distribution and branch content, but different branch length ranging from two to six. Okabe et al. [14] examined the sol-gel transitions of LLDPE, which are copolymers with various comonomers from butene to octene. Comparisons with experimental results from literatures $[11,14]$ are shown in Figure 9. The

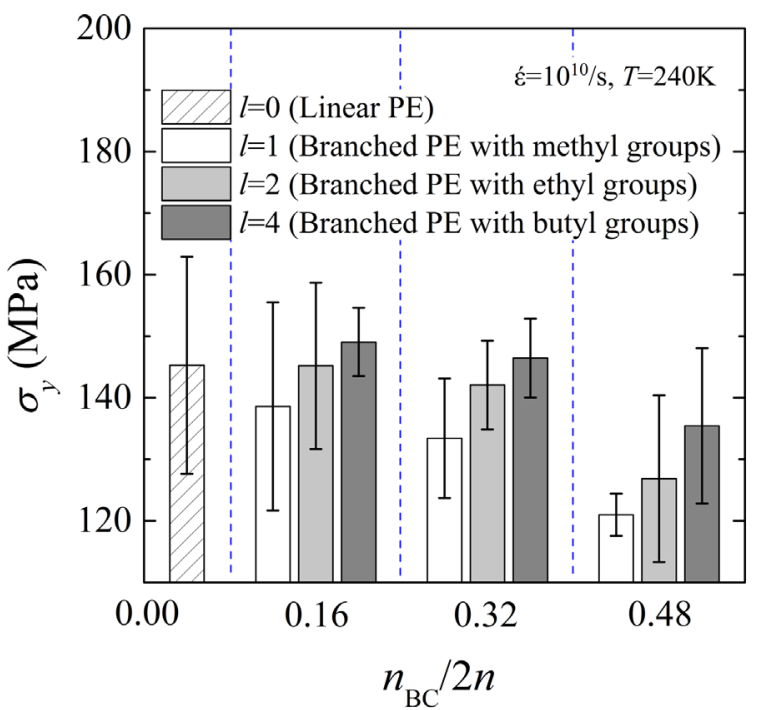

Figure 8. (Colour online) Yield strengths of PE systems subject to a tensile loading with $\varepsilon=10^{10} / \mathrm{s}$ at $240 \mathrm{~K}$.

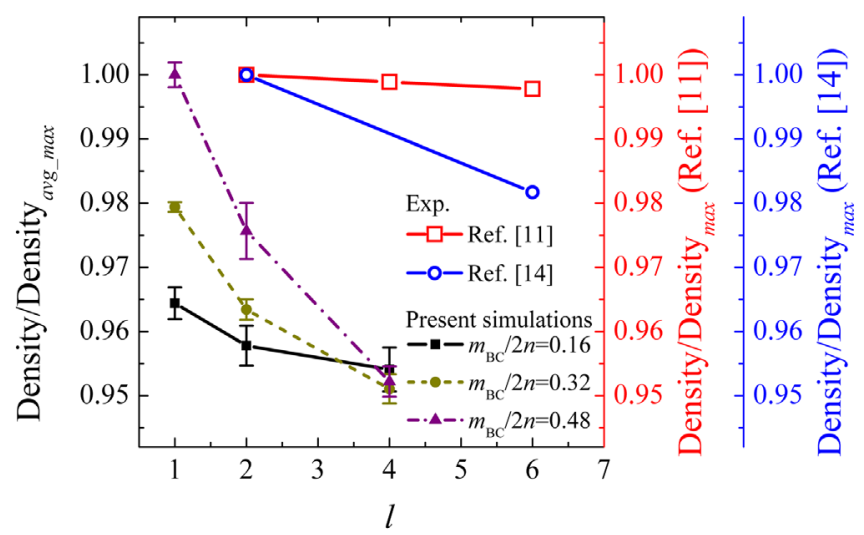

Figure 9. (Colour online) Comparison of branch length effect on equilibrium density between present simulation and existing experimental measurements.

ordinates are normalised densities by maximum values, which are denoted as max in subscript. Especially, the present results are normalised with respect to the maximum value of average equilibrium density with a subscript avg_max. Inversely proportional relationship between the branch length and the equilibrium density is observed both in current simulation and available experimental data, even though it shows a slight deviation due to different systems in microstructures.

\subsubsection{Dynamic responses}

Jordan and Brown [4] examined the dynamic compressive response of LDPE as a function of strain rate using split Hopkinson pressure bar (SHPB) experimentally. They mentioned that molecular conformation, volume fraction of crystallinity and strain rate govern the material responses. Hossain et al. [8] carried out MD simulations on deformation mechanisms of amorphous PE using linear UA models. As shown in Figure 7, stress-strain behaviour experiences elastic, yield, strain softening and strain hardening regions, which is also observed in previous experiments and simulations. In addition, the characterisation 


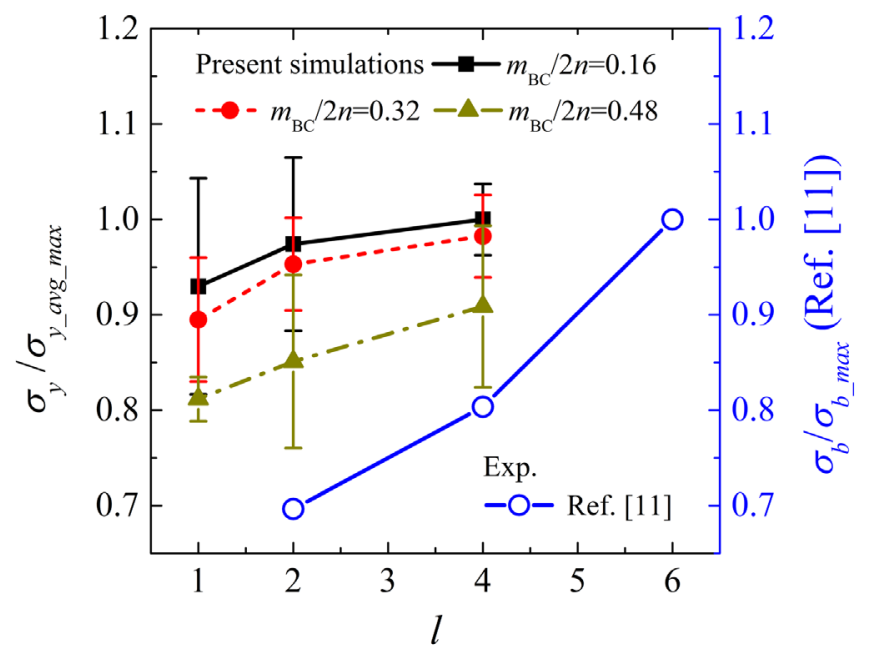

Figure 10. (Colour online) Comparison of branch length effect on dynamic strength between current simulation and available experimental data.

of the strain rate dependence described in literatures is in an agreement with current simulation as shown in Figure 7.

Mortimer [12] held the opinion that the increase in amount of short-chain branching in PE results in yield tensile strength decreasing. The similar statement was also expressed by Zhang et al. [40]. These available results provide a validation of current results as shown in Figure 8.
Figure 10 plots the dynamic strength of present simulation and existing experimental data. Yield strength $\sigma_{y}$ in preset work and fracture strength $\sigma_{b}$ in existing literature [11] were chosen to represent dynamic strength. The ordinate is normalised yield stress $\sigma_{y \text { avg } \max }$ for current simulation and normalised brittle fracture tensile stress $\sigma_{b \text { max }}$ for experimental measurements [11], respectively. It can be observed that $\mathrm{PE}$ with longer branch length holds higher dynamic strength with a given branch content. The simulation shows a good agreement with the experimental results carried out by Liu and Baker [11]. They provided two possible explanations for the increase in the dynamic strength as the branch length increases. One is that the process of PE molecular chain folding into a growing crystal lamella is restricted by the increased length of a chain branching, which increases the number of inter-lamellar tie-molecules. The other is that the increase in the content of the second soft phase resulting from the increased length of chain branching contributes to improve the dynamic strength.

The proportional relation between impact energy and branch length was confirmed by the experimental results [41]. In addition, Gupta et al. [42] carried out the experiments on copolymers of ethylene with 1-butene, 1-hexene and 1-octene to examine the effect of chain length. They found that the tensile strength is proportional to the branch length at high deformation rate (ca. $1 \mathrm{~m} / \mathrm{s}$ ) even though it holds as a constant at slower deformation rates (up to $510 \mathrm{~mm} / \mathrm{min}$ ). The dart impact strength of samples (a)

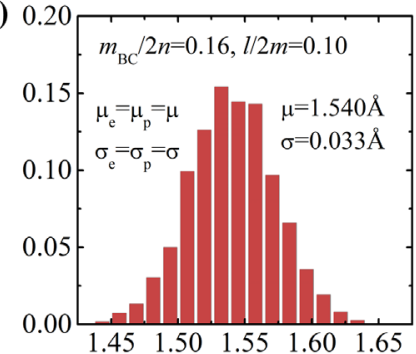

(b)

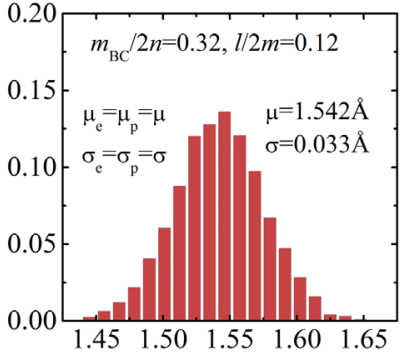

(c)

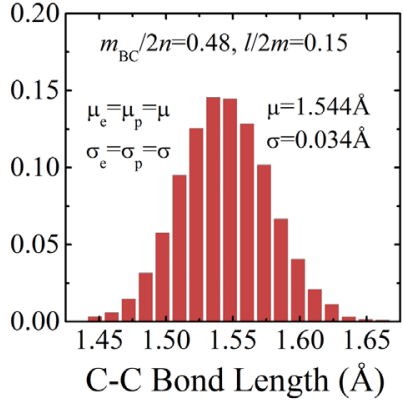

Branched PE with butyl groups, $l=4$

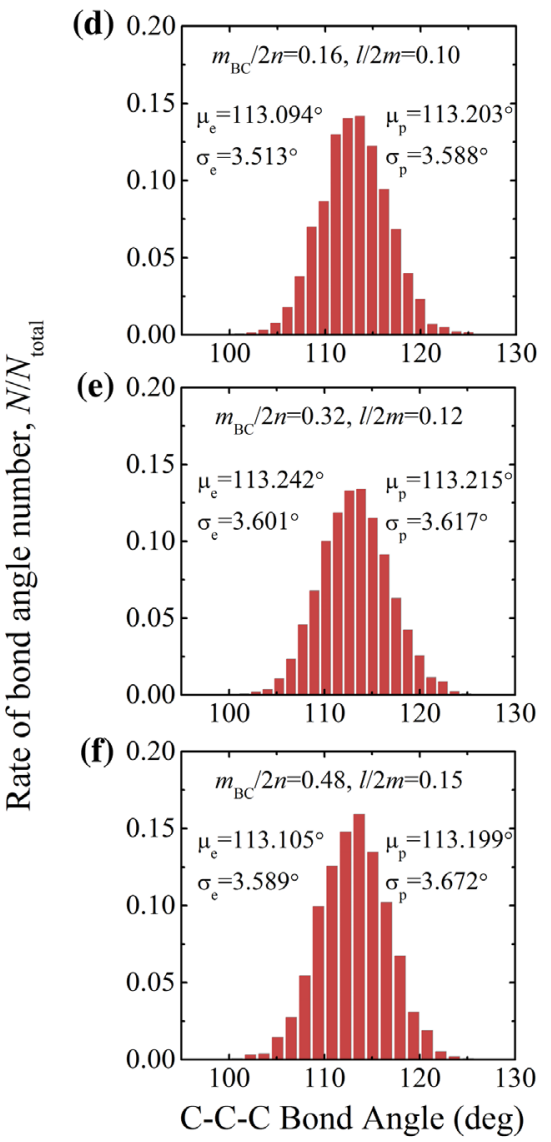

at equilibrium

at stress peak

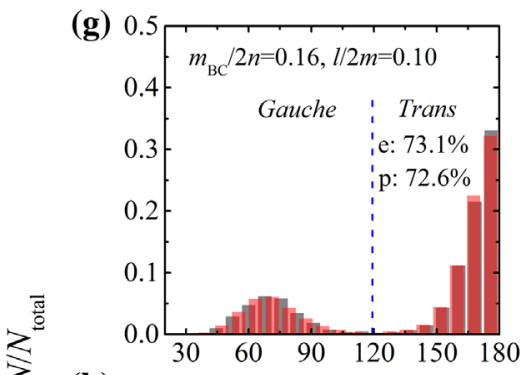

(h)

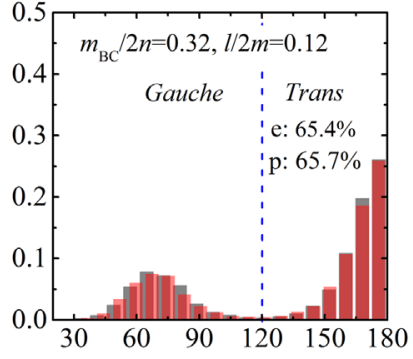

(i) 0.5

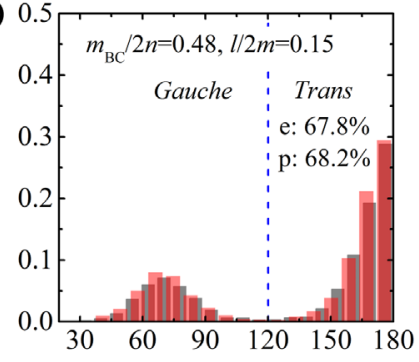

C-C-C-C Dihedral Angle (deg)

Figure 11. (Colour online) Distributions of molecular structural components at equilibrium and stress peak $\left(\dot{\varepsilon}=10^{10} / \mathrm{s}\right)$ for branched PE with butyl groups. 
Branched PE with butyl groups, $l=4$

(a) at equilibrium

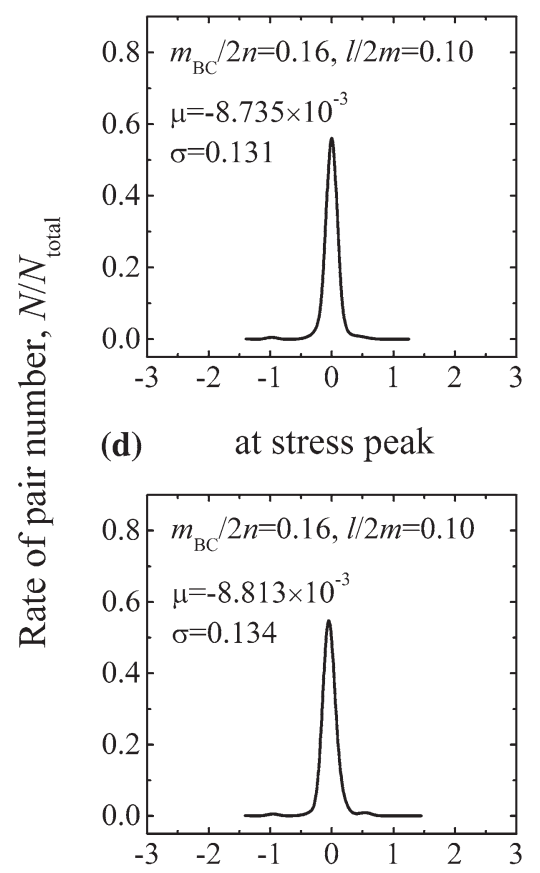

(b) at equilibrium

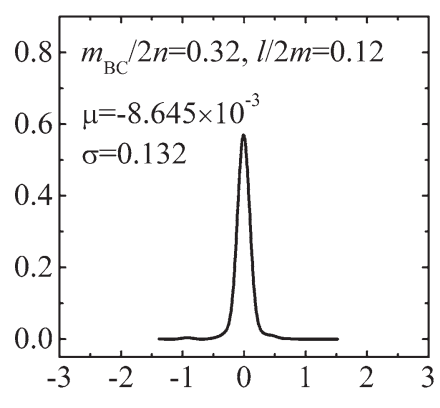

(e) at stress peak

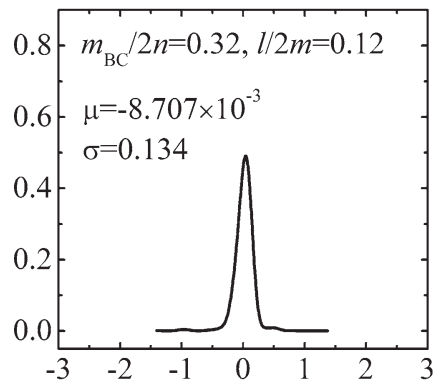

C-C Pair Energy (kcal/mol) (c) at equilibrium

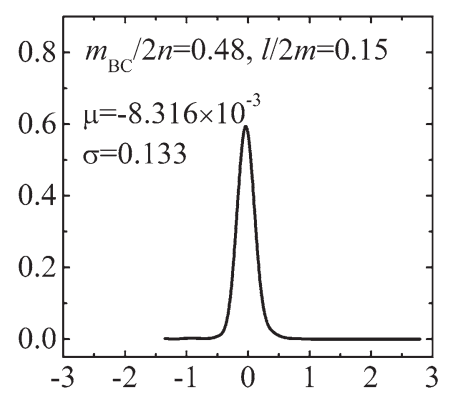

(f) at stress peak

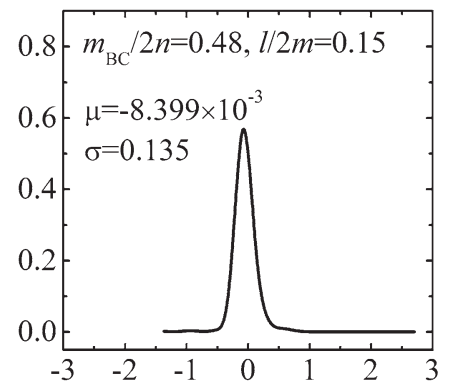

Figure 12. Statistics of $C-C$ pair energy of branched $P E$ with butyl groups at equilibrium and stress peak.

with 1-octene and 1-hexene was also observed to be higher than that those with 1-butene.

Generally, the simulation illustrates a good agreement with available experimental data. It fully supports the reliability of current method to capture the mechanical properties of PE to provide atomistic descriptions.

\section{Discussions}

As illustrated in Figure 11, the microstructures of branched PE with butyl groups were characterised by the distributions of $\mathrm{C}-\mathrm{C}$ bond length (left column), $\mathrm{C}-\mathrm{C}-\mathrm{C}$ bond angle (middle column) and $\mathrm{C}-\mathrm{C}-\mathrm{C}-\mathrm{C}$ dihedral angle (right column) at equilibrium (grey bar with a subscript e) and first stress peak $\left(\dot{\varepsilon}=10^{10} / \mathrm{s}\right.$ in tensile, translucent red bar with a subscript $\mathrm{p}$ ), respectively. The chain geometry of branched PE with butyl groups was normalised with respect to the total number of each component as described in each ordinate. $\mu$ and $\sigma$ are the average value and standard deviation of each component, respectively.

The percentages of trans conformation in dihedral distributions (right column in Figure 11) can be calculated by adopting a threshold as $120^{\circ}$ [8]. As the number of dihedral angle (higher than $120^{\circ}$ ) increases, the inner molecular chains become straighter in terms of gauche-trans transition [43]. It can be assumed that the lower degree of branching allows easier for chains to rotate to trans conformations. However, owing to the formation of amorphous microstructure during relaxation, the inter-branches interactions nearby (branches clustered one by one along the backbone as exemplified) will be enhanced as the degree of branching and branch length increase. On the other hand, a branched system with dispersion distribution eliminates this kind of inter-branches interactions nearby. In this study, the branches were created randomly, which is difficult to avoid the occurrence of successive branches. The effects of branching distributions should be taken into account in the future work.

The average values of $\mathrm{C}-\mathrm{C}$ bond length $\mu$ approximate to 1.540 $\AA$ (Figure 11(a)-(c)). Those of C-C-C bond angle approximate to $113.1^{\circ}$ (Figure $11(\mathrm{~d})-(\mathrm{f})$ ). These values are slightly higher than the equilibrium potential parameters $K_{r}(1.529 \AA)$ and $\theta_{r}$ $\left(112.7^{\circ}\right)$. The similar distributions are obtained in pervious MD simulations [44]. Comparing with the information described in Figure 11 between at equilibrium and first stress peak, the distributions of bond length and bond angle remain approximately constant. However, the rotations of chains to trans conformation changes non-monotonically. On the one hand, previous study [8] suggested that the first stress peak is affected primarily by the non-bonded interactions between chains. They thought the role of bond length or bond angle during elastic deformation is not vital, which is also confirmed in the present simulation as shown in Figure 11(a)-(f). In addition, it is assumed that the distribution of dihedral angles plays an important role in the deformation of PE, as shown in Figure 11(g)-(i).

Omar et al. [1] and Shergold et al. [45] believed that the deformation of polymer chains is restricted by the stretching and bending of the chemical bond subjected to a high strain rate, which also enhances the formation of additional interactions between structures. As a semi-crystalline material, $\mathrm{PE}$ is known as molecular networks including an amorphous phase 
comprising entangled chains with the randomly oriented crystallite phase as physical crosslinks [4]. Hossain et al. [8] held the opinion that the reason of fracture in polymer is chain disentanglement rather than chain scission. In addition, they also found that the majority contribution of total energy increase is the non-bonded energy, which associates with chain slippage mechanisms in the elastic and strain softening region. Sequentially, potential energy acting between the pair of carbon atoms (C-C pair) should be evaluated to demonstrate the entanglement and slippage status partly.

Distributions of $\mathrm{C}-\mathrm{C}$ pair energy at equilibrium (Figure 12(a)-(c)) and first stress peak (Figure 12(d)-(f)) of branched PE with butyl groups are illustrated in Figure 12, where $\mu$ and $\sigma$ are the average value and standard deviation of $\mathrm{C}-\mathrm{C}$ pair energy, respectively. The abscissa is $\mathrm{C}-\mathrm{C}$ pair energy and the ordinate represents the normalised $\mathrm{C}-\mathrm{C}$ pair numbers with respect to the total ones. The potential energies of most $\mathrm{C}-\mathrm{C}$ pairs distribute near zero. Smaller $\mu$ at stress peak is the result of the deformation under tension. In addition, the proportional relation of $\mu$ and $m_{\mathrm{BC}} / 2 n$ both at equilibrium and stress peak provide a support to assume that load-bearing capacity of branched PE with butyl groups increases as branch content decreases from pair energy point of view.

As shown in Figure 6, the effect of branch content is not strict monotonic for the branched PE with butyl groups, which probably depends on the influence of chain branching distribution mentioned above. As a complement of the parameter $m_{\mathrm{BC}} / 2 n$, the proportion of branch length $l$ in the length of backbone $2 m$ should be taken into account, which is listed in the last column of Table 1. Strict monotonic effect of branch content $m_{\mathrm{BC}} / 2 n$ can be obtained when the ratio of $l$ to $2 m$ less than 0.10 .

Big differences between linear PE and branched one can be obtained as shown in Figures 5, 6 and 8. It is necessary to evaluate the reliability of adopting linear PE for simplicity in simulations.

\section{Conclusions}

The effects of length and content of chain branching on the mechanical properties of $\mathrm{PE}$ were evaluated using MD simulations in atomic-scale. Three types of branched chains, methyl, ethyl and butyl groups, were adopted to distribute along the backbone. The reliability of current simulation was validated through comparisons with previous experimental results. The characteristic of strain rate dependence was captured in the plastic flow deformation of PE by providing a uniaxial tensile loading with a given strain rate. There are big differences in mechanical properties of linear PE and branched one. The results show that the length of chain branching is inversely proportional to the equilibrium density of branched PE systems. The effect of branch length on equilibrium density becomes more significant for higher degree of branching. On the contrary, the relationship between the branch length and the yield strength of branched $\mathrm{PE}$ systems is proportional. With the prerequisite of the ratio of branch length to the backbone length less than 0.10 , the content of chain branching $m_{\mathrm{BC}} / 2 n$ is strictly proportionate to the equilibrium density and inverse-proportional to the average value of yield strength $\sigma_{y}$ of branched PE systems. The important role of the distribution of dihedral angles in the deformation of PE should be paid attention. The effect of the non-bond interactions on the load-bearing capacity is significant. It is assumed that the distribution of chain branching affects the performances of PE to some extent, which is worth further discussing.

\section{Acknowledgements}

The authors wish to acknowledge the support from the National Natural Science Foundation of China. The computations were supported by the Computing Facility, Institute of Mechanics, Chinese Academy of Sciences.

\section{Disclosure statement}

No potential conflict of interest was reported by the authors.

\section{Funding}

This work was supported by the National Natural Science Funds of China under [grant number 11672314].

\section{ORCID}

Lijuan Liao (D) http://orcid.org/0000-0003-1753-6373

\section{References}

[1] Omar MF, Akil HM, Ahmad ZA. Effect of molecular structures on dynamic compression properties of polyethylene. Mater Sci Eng A. 2012;538:125-134.

[2] Vicente-Alique E, Vega JF, Robledo N, et al. Study of the effect of the molecular architecture of the components on the melt rheological properties of polyethylene blends. J Polym Res. 2015;22:1-11.

[3] Ramachandran R, Beaucage G, Kulkarni AS, et al. Persistence length of short-chain branched polyethylene. Macromolecules. 2008;41:9802-9806.

[4] Jordan JL, Casem DT, Bradley JM, et al. Mechanical properties of low density polyethylene. J Dyn Behavior Mater. 2016;2:411-420.

[5] Zhang R, Guo H, Lei J, et al. Effect of molecular structure on the performance of polyacrylic acid superplasticizer. J Wuhan Univer Technol Mater Sci Ed. 2007;22:245-249.

[6] Kalyon DM, Yu DW, Moy FH. Rheology and processing of linear low density polyethylene resins as affected by alpha-olefin comonomers. Polym Eng Sci. 1988;28:1542-1550.

[7] Yan D, Wang WJ, Zhu S. Effect of long chain branching on rheological properties of metallocene polyethylene. Polymer. 1999;40:1737-1744.

[8] Hossain D, Tschopp MA, Ward DK, et al. Molecular dynamics simulations of deformation mechanisms of amorphous polyethylene. Polymer. 2010;51:6071-6083.

[9] Dartora PC, Santana RMC, Moreira ACF, et al. The influence of long chain branches of LLDPE on processability and physical properties. Polímeros. 2015;25:531-539.

[10] Kline DE, Sauer JA, Woodward AE. Effect of branching on dynamic mechanical properties of polyethylene. J Polym Sci. 1956;XXII:455462.

[11] Liu TM, Baker WE. The effect of the length of the short chain branch on the impact properties of linear low density polyethylene. Polym Eng Sci. 1992;32:944-955.

[12] Mortimer GA. The effect of short-chain branch structure on the properties of low-density polyethylene. J Appl Polym Sci. 1971;15:1231-1235.

[13] Moyassari A, Mostafavi H, Gkourmpis T, et al. Simulation of semicrystalline polyethylene: effect of short-chain branching on tie chains and trapped entanglements. Polymer. 2015;72:177-184.

[14] Okabe M, Isayama M, Matsuda H. Sol-gel transitions of linear low density polyethylenes in organic solvents. Polym J. 1985;17:369-376.

[15] Raumann G, Saunders DW. The anisotropy of Young's modulus in drawn polyethylene. Proc Phys Soc. 1961;77:1028-1037. 
[16] Chiou S-T, Cheng W-C, Lee W-S. Strain rate effects on the mechanical properties of a $\mathrm{Fe}-\mathrm{Mn}-\mathrm{Al}$ alloy under dynamic impact deformations. Mater Sci Eng A. 2005;392:156-162.

[17] Fu S, Wang Y, Wang Y. Tension testing of polycarbonate at high strain rates. Polym Testing. 2009;28:724-729.

[18] Omar MF, Akil HM, Ahmad ZA. Static and dynamic compressive properties of mica/polypropylene composites. Mater Sci Eng A. 2011;528:1567-1576.

[19] Kulmi U, Basu S. A molecular dynamics study of the failure modes of a glassy polymer confined between rigid walls. Modell Simul Mater Sci Eng. 2006;14:1071-1093.

[20] Clarke JHR, Brown D. Molecular dynamics modelling of polymer materials. Mol Simul. 1989;3:27-47.

[21] Weiner SJ, Kollman PA, Case DA, et al. A new force field for molecular mechanical simulation of nucleic acids and proteins. J Am Chem Soc. 1983;106:765-784.

[22] Weiner SJ, Kollman PA, Nguyen DT, et al. An all atom force field for simulations of proteins and nucleic acids. J Comput Chem. 1986;7:230-252.

[23] Jorgensen WL, Tiradorives J. The OPLS [optimized potentials for liquid simulations] potential functions for proteins, energy minimizations for crystals of cyclic peptides and crambin. J Am Chem Soc. 1988;110:1657-1666.

[24] Jorgensen WL, Maxwell DS, Tirado-Rives J. Development and testing of the OPLS all-atom force field on conformational energetics and properties of organic liquids. J Am Chem Soc. 1996;118:11225-11236.

[25] Sun H. Force field for computation of conformational energies, structures, and vibrational frequencies of aromatic polyesters. J Comput Chem. 1994;15:752-768.

[26] Sun H. COMPASS: an $a b$ initio force-field optimized for condensedphase applications overview with details on alkane and benzene compounds. J Phys Chem B. 1998;102:7338-7364.

[27] Humphrey W, Dalke A, Schulten K. VMD: visual molecular dynamics. J Mol Graph. 1996;14:33-38.

[28] Jewett A. Moltemplate Manual. Jensen Lab (Caltech), Shea Lab (UCSB): Jensen Lab (Caltech), Shea Lab (UCSB). 2016 Dec 8.

[29] Plimpton S. Fast parallel algorithms for short-range molecular dynamics. J Comput Phys. 1995;117:1-19.

[30] Nikkhah SJ, Moghbeli MR, Hashemianzadeh SM. Dynamic study of deformation and adhesion of an amorphous polyethylene/graphene interface: a simulation study. Macromol Theory Simul. 2016;25:533549.

[31] Yang SR, Gao F, Qu JM. A study of highly crosslinked epoxy molding compound and its interface with copper substrate by molecular dynamic simulations. In: 2010 Electronic Components and Technology Conference; 2010. p. 128-134; Las vegas, NV, USA.
[32] Mahajan DK, Basu S. On the simulation of uniaxial, compressive behavior of amorphous, glassy polymers with molecular dynamics. Int J Appl Mech. 2010;02:515-541.

[33] Yu KQ, Li ZS, Sun JZ. Polymer structures and glass transition: a molecular dynamics simulation study. Macromol Theory Simul. 2001;10:624-633.

[34] Teraoka I. Polymer solutions: an introduction to physical properties. New York, NY: John Wiley \& Sons, Inc.; 2002.

[35] Theodorou DN, Suter UW. Detailed molecular structure of a vinyl polymer glass. Macromolecules. 1985;18:1467-1478.

[36] Kremer K, Grest GS. Dynamics of entangled linear polymer melts: A molecular-dynamics simulation. J Chem Phys. 1990;92:5057-5086.

[37] Adnan A, Sun CT. Effect of adhesive thickness on joint strength: a molecular dynamics perspective. J Adhes. 2008;84:401-420.

[38] Tschopp MA, Bouvard JL, Ward DK, et al. Atomic scale deformation mechanisms of amorphous polyethylene under tensile loading. The Minerals, Metals \& Materials Society (TMS) 2011 Conference Proceedings, Supplemental Proceedings: Materials Fabrication, Properties, Characterization, and Modeling; 2011. p. 789-794; San Diego, CA, USA.

[39] Vu-Bac N, Lahmer T, Keitel H, et al. Stochastic predictions of bulk properties of amorphous polyethylene based on molecular dynamics simulations. Mech Mater. 2014;68:70-84.

[40] Zhang XM, Elkoun S, Ajji A, et al. Oriented structure and anisotropy properties of polymer blown films: HDPE, LLDPE and LDPE. Polymer. 2004;45:217-229.

[41] Yeh JT, Chen BJ, Lee HM. Impact properties of carbon fiber reinforced linear and short-chain branched polyethylenes. Polym Bull. 1994;33:607-614.

[42] Gupta P, Wilkes GL, Sukhadia AM, et al. Does the length of the short chain branch affect the mechanical properties of linear low density polyethylenes? An investigation based on films of copolymers of ethylene/1-butene, ethylene/1-hexene and ethylene/1octene synthesized by a single site metallocene catalyst. Polymer. 2005;46:8819-8837.

[43] Yashiro K, Ito T, Tomita Y. Molecular dynamics simulation of deformation behavior in amorphous polymer: nucleation of chain entanglements and network structure under uniaxial tension. Int J Mech Sci. 2003;45:1863-1876.

[44] Kirichenko AF, Sahu A, Filippov TV, et al. Effects of temperature and strain rate on the deformation of amorphous polyethylene: a comparison between molecular dynamics simulations and experimental results. Modell Simul Mater Sci Eng. 2013;21:065016.

[45] Shergold OA, Fleck NA, Radford D. The uniaxial stress versus strain response of pig skin and silicone rubber at low and high strain rates. Int J Impact Eng. 2006;32:1384-1402. 